\title{
Charge transfer and dd excitations in transition metal oxides
}

\author{
q-dependent dielectric function study by inelastic $X$-ray scattering and optical reflectivity \\ measurements
}

\author{
N. Hiraoka ${ }^{1, a}$, H. Okamura ${ }^{2}$, H. Ishii ${ }^{1}$, I. Jarrige ${ }^{1, b}$, K.D. Tsuei ${ }^{1}$, and Y.Q. Cai ${ }^{1, c}$ \\ 1 National Synchrotron Radiation Research Center (NSRRC), 30076 Hsinchu, Taiwan \\ 2 Graduate School of Science and Technology, Kobe University, 657-8501 Kobe, Japan
}

Received 1st February 2009 / Received in final form 12 May 2009

Published online 16 June 2009 - (c) EDP Sciences, Società Italiana di Fisica, Springer-Verlag 2009

\begin{abstract}
Electronic excitations in transition-metal oxides $\mathrm{MnO}, \mathrm{FeO}, \mathrm{CoO}$ and $\mathrm{NiO}$ are investigated by inelastic X-ray scattering and optical reflectivity measurements. The dielectric functions are derived from the experimental data as a function of the momentum transfer, $q$. Based on the derived $q$-dependent dielectric functions, two types of the charge transfer excitations, i.e., dipolar and non-dipolar charge transfer, are clearly identified. We show that the Mott gaps around $5 \mathrm{eV}$ are defined by the former whereas the latter occurs at higher energies of 8-12 eV. Based on a molecular orbital analysis, we associate the dipolar and the non-dipolar excitations with non-local charge transfer and conventional charge transfer, respectively. These types of excitations are shown to be common for the $3 d$ metal monoxides. On the other hand, the $d d$ excitations observed in $\mathrm{NiO}$ and $\mathrm{CoO}$ at energy $<4 \mathrm{eV}$ do not appear in $\mathrm{FeO}$ and $\mathrm{MnO}$. The reasons are addressed in this report.
\end{abstract}

PACS. 71.20.-b Electron density of states and band structure of crystalline solids - 78.70.Ck X-ray scattering

\section{Introduction}

Insulators having a band gap due to electron correlation, the so-called Mott insulators, were formerly classified into either the Mott-Hubbard type or the charge transfer type [1-4]. Correlated $d$ electrons in this scheme split into two bands, i.e., the upper Hubbard band (UHB) and the lower Hubbard band (LHB): if the LHB is closer to the Fermi level than the ligand $p$ band, the system is the Mott-Hubbard type, in which the conductive gap (Mott gap) is defined as the transition from the LHB to the UHB; the system is otherwise the charge transfer type, in which the Mott gap is given by the transition from the $p$ band to the UHB. This concept was widely accepted for two decades after its introduction for the interpretation of a large volume of photoemission data (see, e.g., Refs. [5-8]). However, many of recent reports adopting advanced theory show that correlated $d$ electrons generally split into three bands, i.e., UHB, LHB, plus an additional band that is well hybridized with the $p$ bands between the UHB and the LHB [9-14]. This third band has been argued to

\footnotetext{
a e-mail: hiraoka@spring8.or.jp

b Present address: Japan Atomic Energy Agency, (JAEA/ SPring-8), 1-1-1, Kouto, Sayo, 6795198 Hyogo, Japan

c Present address: National Synchrotron Light Source II, Brookhaven National Laboratory, Upton, 11973 NY, USA
}

correspond to a Zhang-Rice singlet in cuprates, implying that the Mott gap is given by the transition from this Zhang-Rice band to the UHB. As prototypes of Mott insulators, the transition-metal monoxides (TMO, in which $\mathrm{TM}=\mathrm{Mn}, \mathrm{Fe}, \mathrm{Co}$, or $\mathrm{Ni}$ ) of $\mathrm{NaCl}$ type remain subject of theoretical and experimental investigations.

An optical absorption spectrum, which is typically obtained from optical reflectivity measurements, is a source of fundamental and important experimental data pertaining to the Mott insulator. The optical reflectivity of $\mathrm{NiO}$ exhibits a sharp maximum around $4 \mathrm{eV}$, i.e., the well known charge-transfer peak defining the Mott gap [15]. $\mathrm{CoO}$ exhibits a similar feature around $5 \mathrm{eV}$ although it is broad. As mentioned above, the details of these excitations are still debated. The reflectivities of $\mathrm{FeO}$ and $\mathrm{MnO}$ over a wide range of energy, which is necessary to obtain the absorption coefficient, and the optical conductivity, or dielectric functions, have not yet been reported despite their fundamental importance (see, Refs. [16,17] for the optical data for partial ranges).

In the present study, we have investigated electronic excitations in TMO using non-resonant inelastic X-ray scattering (IXS) and optical reflectivity measurements. IXS is a recent method developed in synchrotron facilities whereas optical measurements are well established. IXS, which detects charge excitations as a function of 
the momentum transfer, $q$, is similar to electron energy loss spectroscopy (EELS) in this sense, but one can also thereby measure the region of large $q$ because of the negligible effect of multiple scattering. A recent nonresonant IXS experiment, conducted by Larson et al. has revealed $d d$ excitations having remarkably large intensities at $q>G$ along [111] in $\mathrm{NiO}$ and $\mathrm{CoO}[18]$. ( $\mathrm{G}$ is the first reciprocal lattice vector, $\sim 25 \mathrm{~nm}^{-1}$ ). Authors of this report focused on $d d$ excitations, and mentioned neither charge transfer excitations nor Mott gaps. IXS has an advantage that its probed length scale is variable as a function of $q$; with this property, IXS is applicable to both inter-atomic excitations such as charge transfer and intraatomic excitations such as $d d$ excitation. We have derived the dielectric functions for $0.5 \sim 300 \mathrm{eV}$ and $0.1 \sim 30 \mathrm{eV}$ at $q=0 \rightarrow(7 / 4) G$ with IXS and optical reflectivity measurements, respectively, so as to investigate excitations of both types.

Resonant IXS (RIXS) is commonly used for studies of transition-metal compounds because the resonance provides greater intensities [19,20], but we show that non-resonant IXS can provide much cleaner information. Derivation of the dielectric functions makes the exact comparison between the IXS data and the optical data possible and the excitation features become more readily identifiable. Our work is thus significant because it is not only a mere study of electronic excitation in TMO but also the demonstration of the unique capability of non-resonant IXS.

\section{Experiments and calculations}

IXS spectra were measured at the Taiwan IXS beamline (BL12XU) in SPring-8 [21] with X-rays of energy $9.888-10.188 \mathrm{keV}$ and resolution $\sim 250 \mathrm{meV}$ from 0.5 to $12 \mathrm{eV}$, and resolution $\sim 1.4 \mathrm{eV}$ from 12 to $300 \mathrm{eV}^{1}$. The beam from an undulator was monochromated with a Si 111 double crystal monochromator. For experiments at resolution $\sim 250 \mathrm{meV}$, a $\mathrm{Si} 400$ channel-cut crystal monochromator was used after the double crystal monochromator. We used three 2-m bent Si 555 analyzers placed along the axis perpendicular to the (horizontal) scattering plane. The corresponding momentum resolutions were $\sim 2.1 \mathrm{~nm}^{-1}$ in the horizontal plane and $\sim 8.4 \mathrm{~nm}^{-1}$ in the vertical plane. $\mathrm{MgO}$ was also measured as a reference: $\mathrm{MgO}$ has a crystal structure of the same type and a similar lattice parameter, but no $d$ electron. Glass was measured to provide a reference of tails of the elastic lines. The optical reflectivities in $0.1 \sim 2 \mathrm{eV}$ were measured with an interferometer in a laboratory whereas those in $2 \sim 30 \mathrm{eV}$ were obtained at the beamline BL7B in the UVSOR synchrotron facility [22]. The energy resolving power was $E / \Delta E \sim 100$, where $E$ and $\Delta E$ are the energy and the energy width, respectively. The samples were obtained from commercial sources. The $q$ vectors and the

\footnotetext{
1 The spectrum from 0.5 to $12 \mathrm{eV}$ of $\mathrm{NiO}$ was measured with
} a resolution $170 \mathrm{meV}$ with X-rays of energy $7.910-7.922 \mathrm{keV}$. polarization vectors were maintained parallel to axis [111] in the IXS and the optical experiments, respectively.

As can be seen later, our data exhibit a rich variety of excitations, including intra-atomic excitations, interatomic excitations, and also possibly inter-cluster excitations showing a $q$ dispersion. Although there are several advanced theories having the potential to fully reproduce the $q$-dependent dielectric functions, they are not readily applicable. In order to be possible, they have to be based on a model fully describing the behaviors of correlated electrons as well as atomic multiplets for the $d$ orbitals. The $q$-dependent features make the application of cluster models difficult, particularly for three-dimensional systems like the NaCl-type TMO because a large number (e.g., several tens) of clusters are required. We have therefore attempted only a simple analysis on a single cluster, aiming for qualitative understanding of the underlying physical principle behind the observed excitations.

We calculated molecular orbitals in $\mathrm{NiO}$ as a representative model of the TMO. One can easily apply similar interpretations to the other compounds. In order to obtain the energies for excitations, a further step such as a multi-configuration calculation, like one by Haverkort et al. [23], is necessary but such an analysis is not made in this study. Nevertheless, knowledge of the molecular orbitals is particularly helpful in understanding the physics behind each excitation. In fact, most of the observed behaviors of the excitation features are reproducible from a simple analysis of the molecular orbitals as is shown later. Molecular orbitals in the $S=1,\left(\mathrm{NiO}_{6}\right)^{10-}$ cluster were calculated based on ab initio unrestricted HartreeFock (UHF) method using the GAMESS package [24]. The cluster was embedded in point charges describing the Madelung potentials, +2 for the $\mathrm{Ni}$ site and -2 for the $\mathrm{O}$ site in the lattice. $\mathrm{NiO}$ is slightly distorted below the Néel temperature, but we assumed non-distorted clusters because the distortion is small. We used $0.2091 \mathrm{~nm}$ for the distance between the $\mathrm{Ni}$ and $\mathrm{O}$ atoms, which was determined from our X-ray diffraction experiments with an accuracy of $+/-0.0005 \mathrm{~nm}$.

\section{Results and discussions}

Figure 1 shows the measured IXS $(q \neq 0)$ and reflectivity spectra $(q=0)$; whereas Figure 2 shows the real $\left(\varepsilon_{1}\right)$ and imaginary $\left(\varepsilon_{2}\right)$ parts of the dielectric functions, obtained with the Kramers-Kronig relation and the $f$-sum rule (see, e.g., Ref. [25]). TMO exhibit many common features, generally of four types, in the $\varepsilon_{2}$ (see Fig. 2): (i) sharp $d d$ excitations within the gap $(<4 \mathrm{eV})$, (ii) broad maxima around $5 \mathrm{eV}$, being prominent at small $q$, (iii) continuous features in $8 \sim 12 \mathrm{eV}$, being noticeable around $q=G$, (iv) a feature showing a large dispersion at energy $>12 \mathrm{eV}$. We discuss each feature in the following.

\section{1 dipolar and non-dipolar charge transfer}

We first discuss the charge transfer excitations, i.e., the feature-(ii) (iii), and (iv). The feature-(ii), seen around 


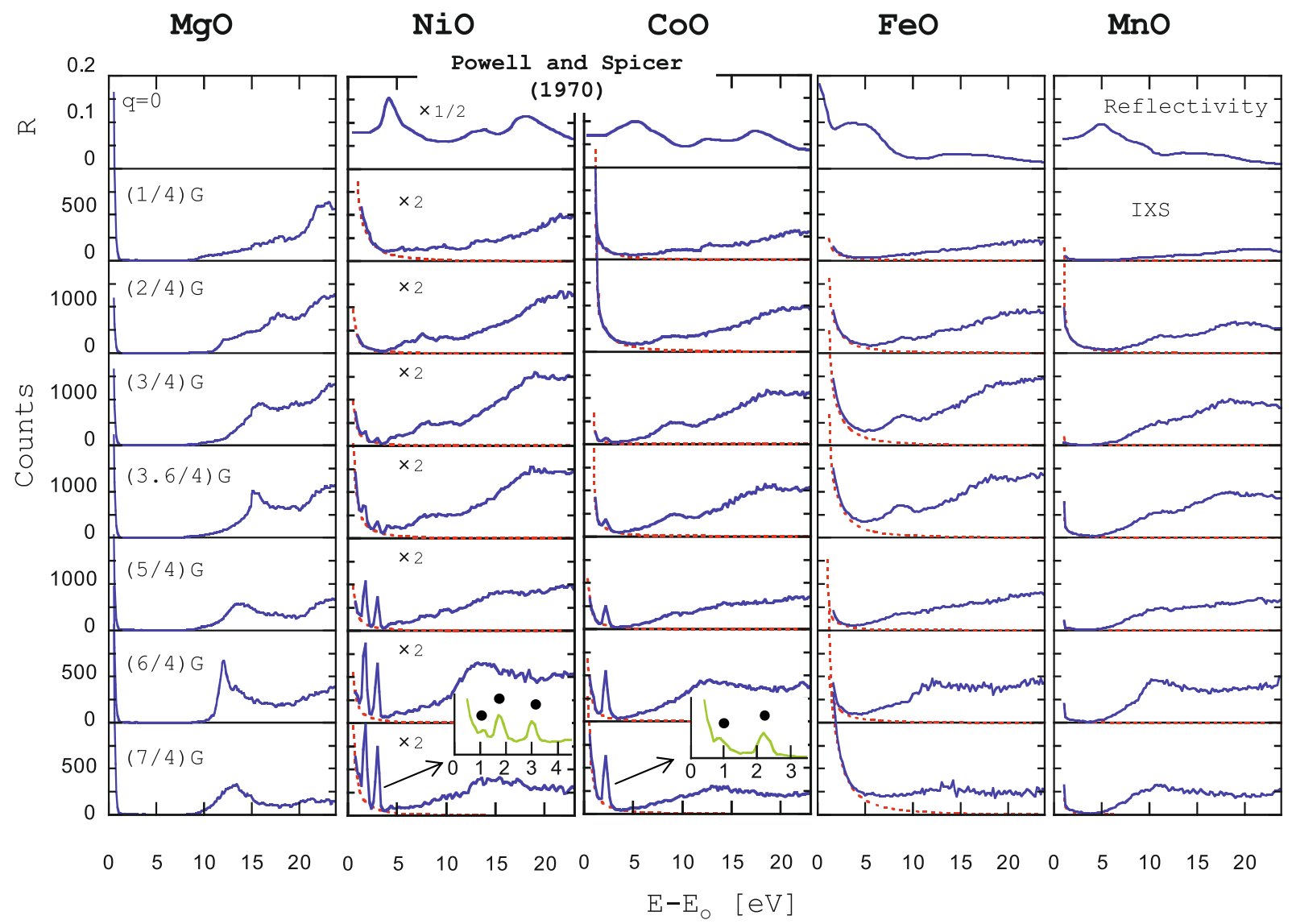

Fig. 1. (Color online) IXS and optical reflectivity spectra: dotted lines denote fitted tails of the elastic lines. The insets provide the details of the $d d$ excitations: three peaks are recognizable in $\mathrm{NiO}$ and two peaks in CoO. For the optical reflectivities $(q=0)$ of $\mathrm{NiO}$ and $\mathrm{CoO}$, the curves by Powell and Spicer [15] are re-plotted. The G refers to the first reciprocal lattice vector, [111]. Its magnitude is given by $25.8,25.8,25.4,25.5$, and $24.5 \mathrm{~nm}^{-1}$ for $\mathrm{MgO}, \mathrm{NiO}, \mathrm{CoO}, \mathrm{FeO}$, and $\mathrm{MnO}$, respectively.

$5 \mathrm{eV}$, exhibits a typical behavior of the dipolar transition: they are prominent in the optical data $(q=0)$ but become rapidly suppressed as $q$ increases (Fig. 2). This feature in $\mathrm{NiO}$ shows a sharp onset at $q=0$ that appears to shift its center of mass to greater energy with a smooth onset at $q=(1 / 2) G$. Around $q=G$, this feature is weak but the sharp onset shifts discernibly back to smaller energy, exhibiting a dispersive behavior. This behavior is difficult to observe for the other oxides because they have no sharp onset.

As is shown later, the $\left(\mathrm{NiO}_{6}\right)^{10-}$ molecular orbital analysis predicts this dipolar charge transfer $(d-\mathrm{CT})$ to be the transition from the $\mathrm{O}_{6} t_{1 u}$ orbitals to the $e_{g}$ orbitals dominated by TM. The $t_{1 u}$ orbitals do not hybridize with the $d$ orbitals of the TM at the center of the cluster $\left(\mathrm{TM}_{i n}\right)$, but can hybridize with the $d$ orbitals of the TM outside the cluster $\left(\mathrm{TM}_{\text {out }}\right)$. The $\mathrm{O} \rightarrow \mathrm{TM}_{\text {in }}$ charge transfer may hence be accompanied by the $\mathrm{TM}_{\text {out }} \rightarrow \mathrm{TM}_{\text {in }}$ charge transfer. This transition corresponds well to the nonlocal charge transfer (NLCT) that Duda et al. suggested in their work on the O K-edge RIXS study [26].

On the other hand, the feature-(iii) seen at $8 \sim 12 \mathrm{eV}$ is not discernible in the optical data but is prominent in the IXS data at $q \sim G$, exhibiting a behavior of non-dipolar transitions. Our cluster analysis described below confirms this excitation to arise from the non-dipolar charge transfer excitation ( $n d-\mathrm{CT}$ ) from the $e_{g}$ orbitals dominated by O to the $e_{g}$ orbitals dominated by TM, i.e., conventional (but non-dipolar) charge transfer from $\mathrm{O}$ to TM.

The features above $12 \mathrm{eV}$ or features-(iv) are similar to those in $\mathrm{MgO}$, but they are more smeared, likely because of correlation effects. They are hence mainly excitations of $p \rightarrow s, s \rightarrow p, s \rightarrow s$, and/or $p \rightarrow p$, in which $d$ orbitals are little involved.

\section{2 dd excitations}

The behaviors of the $d d$ excitations or features-(i) are basically the same as those described in the report of IXS on $\mathrm{NiO}$ and $\mathrm{CoO}$ by Larson et al. [18]. The $d d$ excitations are not discernible in the optical data, but they become abruptly enhanced as $q$ increases, indicating the localized nature of the excitations (Fig. 1). An additional fact found in this study is that the $d d$ excitations are not observed in $\mathrm{FeO}$ and $\mathrm{MnO}$, likely for different reasons. $\mathrm{MnO}$ has totally filled up-spins and totally empty down-spins $\left(d \uparrow^{5} \downarrow^{0}\right)$; as spin-flip transitions are negligible in IXS, there is almost no possibility of a $d d$ excitation, in sharp contrast to 


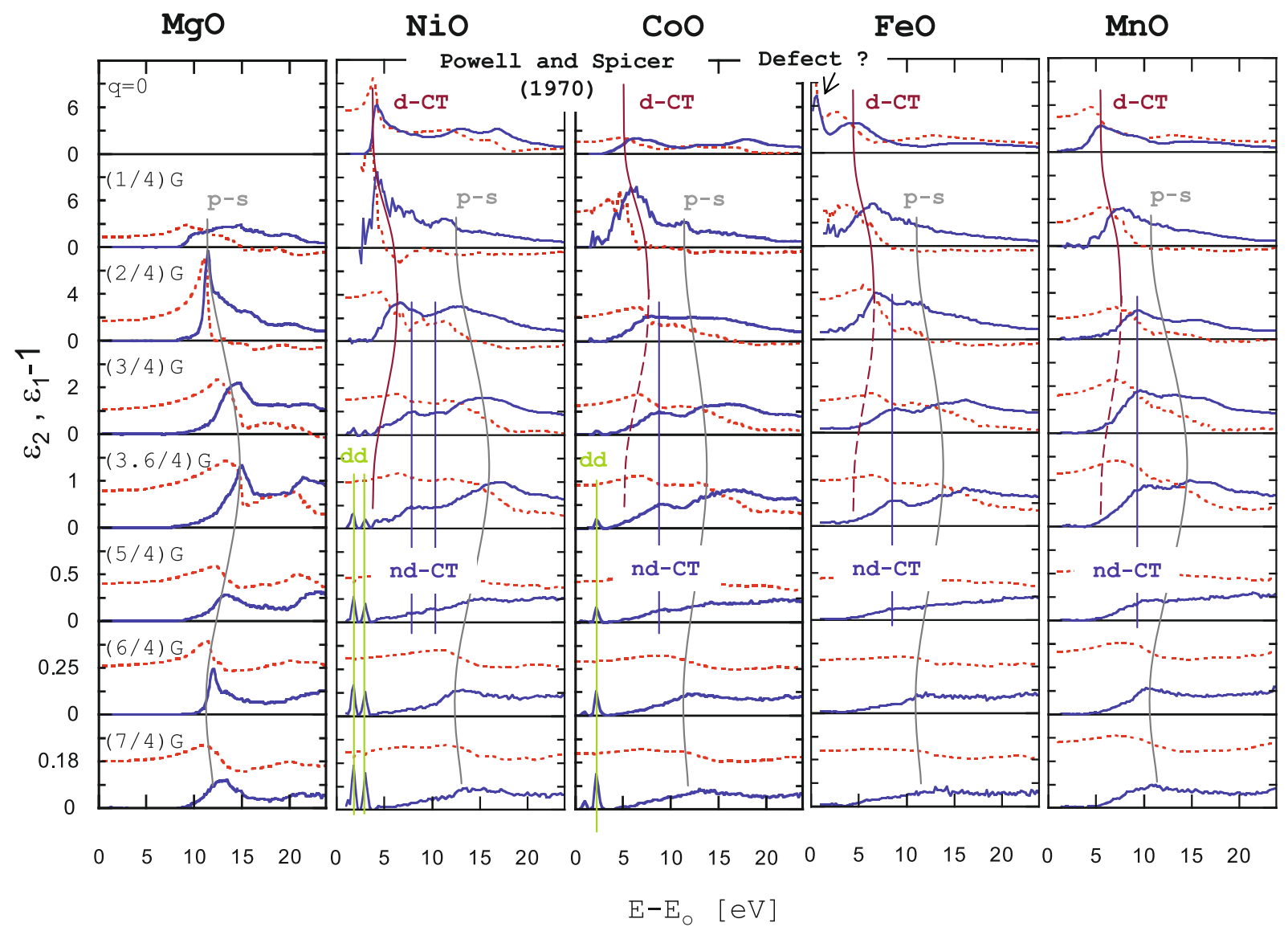

Fig. 2. (Color online) Real parts $\left(\varepsilon_{1}\right.$, broken curves) and imaginary parts $\left(\varepsilon_{2}\right.$, solid curves) of dielectric functions : $d d, d$-CT, and $n d$-CT refer to $d d$ excitations, dipolar charge transfers, and non-dipolar charge transfer excitations, respectively. The $p-s$ denotes the excitations from $p \rightarrow s, s \rightarrow p, s \rightarrow s$, or $p \rightarrow p$ orbitals. For $\mathrm{NiO}$ and $\mathrm{CoO}$ at $q=0$, the curves by Powell and Spicer [15] are re-plotted. The $\mathrm{G}$ refers to the first reciprocal lattice vector, [111].

the Mn M-edge RIXS for which many $d d$ excitations are reported [27]. On the other hand, FeO $\left(d \uparrow^{5} \downarrow^{1}\right)$ has many possibilities of $d d$ excitations, but they may be masked by a band below $2 \mathrm{eV}$, which is clearly seen in the reflectivity spectrum. The nature of this band is unclear but it is likely caused by non-stoichiometry: FeO is well known to be prone to defects, and this property is manifested by the intense elastic lines in the IXS data (Fig. 1).

Unlike the previous report [18], three maxima are recognizable for the $d d$ excitations in $\mathrm{NiO}$ although the $1 \mathrm{eV}$ feature is weak (see, Fig. 1 inset). This result is consistent with RIXS measurements on the Ni M-edge [28], whereas Haverkort et al. predict the $1 \mathrm{eV}$ peak to be forbidden along [111] in the non-resonant case [23]. The fact that three analyzers we used have a large $q$ acceptance along the axis perpendicular to the scattering plane explains our observations.

\subsection{UHF molecular orbitals}

As is mentioned above, to obtain the molecular orbitals, we adopt the $\left(\mathrm{NiO}_{6}\right)^{10-}$ cluster as the minimum model reproducing the charge transfers and the $d d$ excitations. The dielectric functions of the TMO consist of several common features. It is therefore essential to interpret those of $\mathrm{NiO}$ showing richer structures than the others. Once the excitations of $\mathrm{NiO}$ are understood, similar interpretations can be applied to all the TMO members. As the calculation is based on a single Slater determinant, one can not expect it to predict the correct energies of the observed transitions because no effect of the final state is considered. This aspect is not trivial even for more advanced calculations such as those by Larson et al. [18]. Our analyses nevertheless reveal fundamental facts allowing us to intuitively understand each excitation.

The UHF calculation provides 23 molecular orbitals for each spins from five $d$ orbitals and $18 p$ orbitals (for six $\mathrm{O}$ atoms), classified into seven types (see Fig. 3a and also the inset in Fig. 3c): (A) antibonding $e_{g}$ molecular orbitals dominated by $\mathrm{Ni}$, (B) antibonding $t_{1 u}$ purely from $\mathrm{O},(\mathrm{C})$ bonding $e_{g}$ dominated by $\mathrm{O},(\mathrm{D})$ antibonding $t_{2 g}$ mainly by $\mathrm{O},(\mathrm{E})$ bonding $t_{1 u}$ purely from $\mathrm{O},(\mathrm{F})$ bonding $t_{2 g}$ mainly by $\mathrm{Ni}$, and $(\mathrm{G})$ others (not shown). In the Hartree-Fock scheme, orbitals-(A) for up spins (occupied) mimic the LHB whereas those for down spins (unoccupied) the UHB. The orbitals for up-spins are totally filled; transitions thus occur only for down spins 
(a)

$\begin{array}{lllllll}\mathrm{Ni}\left(+\mathrm{O}_{6}\right) e_{g} & O_{6} t_{1 u} & O_{6}(+N i) e_{g} & O_{6}(+N i) t_{2 g} & O_{6} t_{1 u} & N i\left(+O_{6}\right) t_{2 g}\end{array}$
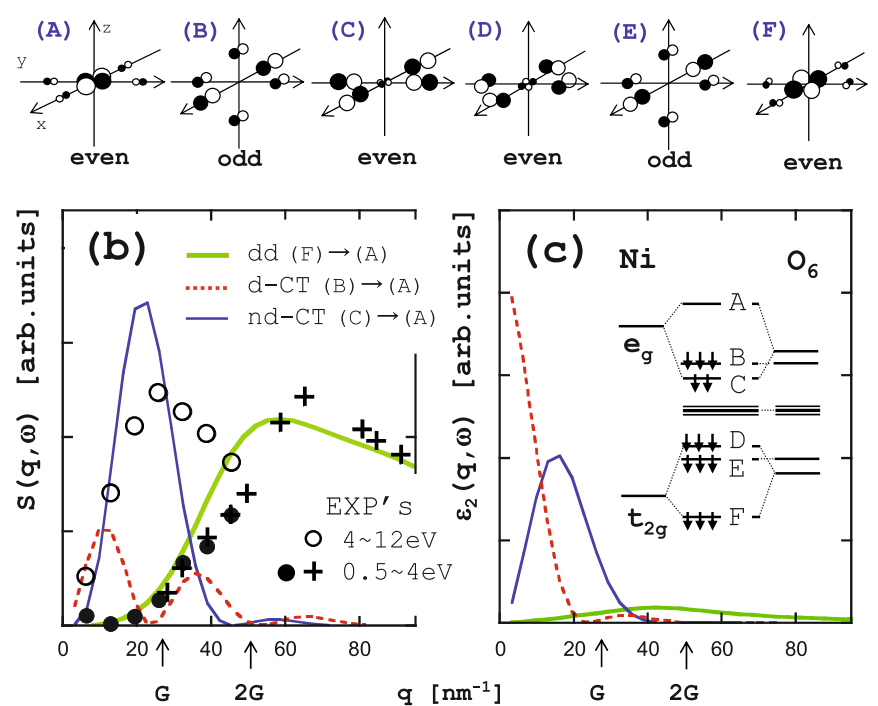

Fig. 3. (Color online) (a) Selected molecular orbitals for each symmetry: $d d$ excitations $(d d)$ are given by $(\mathrm{F}) \rightarrow(\mathrm{A})$; dipolar charge transfer $(d-\mathrm{CT})$ and non-dipolar charge transfer $(n d-\mathrm{CT})$ excitations are dominated by $(\mathrm{B}) \rightarrow(\mathrm{A})$ and $(\mathrm{C}) \rightarrow(\mathrm{A})$, respectively. (b) Calculated IXS scattering cross sections, compared with experiment: $(\bullet)$ and (०) are integrated intensities of experimental spectra at $0.5-4.0 \mathrm{eV}$ and 4.0-12.0 eV, respectively. ( $\circ$ ) include both $d$-CT and $n d-\mathrm{CT}$ as they are not clearly resolved in the experimental data. $(+)$ are the same as $(\bullet)$ but are from another data-set (not shown). (c) Calculated imaginary parts of the dielectric functions: the inset shows the energy diagram for the down spins in $\mathrm{NiO}$.

once spin-flipping is neglected. The intensities of scattering are readily calculated by numerically integrating $\langle f|\exp (i \mathbf{q r})| i\rangle$, in which $|i\rangle$ is an initial state and $\langle f|$ is a final state, which invariably involves orbitals-(A). We calculated the transition matrices for all possible transitions. The followings are transitions that have appreciable intensities: (i) orbitals- $(\mathrm{F}) \rightarrow(\mathrm{A})$, providing excitations localized in $\mathrm{Ni}$ atoms, which correspond to the $d d$ excitations. (ii) orbitals- $(\mathrm{B}) \rightarrow(\mathrm{A})$, corresponding to the $d$-CT (also partly from $(\mathrm{E}) \rightarrow(\mathrm{A})$ but small contributions because of smaller dipole moments) - There is no other channel providing dipolar transitions; (iii) orbitals- $(\mathrm{C}) \rightarrow(\mathrm{A})$, corresponding to the $n d-\mathrm{CT}$.

Figures $3 \mathrm{~b}$ and $3 \mathrm{c}$ are the calculated intensities of scattering or the dynamical structure factors, $\mathrm{S}(q, \omega)$, and the $\varepsilon_{2}$. To calculate $\mathrm{S}(q, \omega)$, we put 1 to $\varepsilon_{1}$ and rescaled $\varepsilon_{2}$ with a common factor to fit to the experimental ones. A reasonable agreement exists with results of experiments in the intensities integrated in several windows of energy. The previous reports $[18,23]$ developed novel theories to reproduce the $q$-evolution of the intensities of the $d d$ excitations based on energy-resolved Wannier functions and configuration interaction clusters, respectively. A comparable reproducibility is obtained with such a simple model for the charge transfer excitations in addition to the $d d$ ones. Note that the previous reports $[18,23]$ only reproduced the latter. The present analysis reproduces also the angular dependence of the intensities of the $d d$ excitations (not shown), which shows a node along [100] in NiO, justifying our interpretation for the observed features of the excitations, and also indicating that our analyses capture the essence in describing the excitations in TMO.

Interestingly, orbitals-(B) $\left[t_{1 u}\right]$, producing the $d$-CT, never hybridize with the central $\mathrm{Ni}$ for the reason of the symmetry. This may be a key distinguishing the non-local charge transfer from the conventional one. Many of recent studies show that the top of the valence band involves considerable contributions from $d$ orbitals (Zhang-Rice band), making our model incompatible with those. This discrepancy is possible because the Zhang-Rice band may arise from the configuration interaction of several Slater determinants. Nevertheless, there is another (or compatible) possibility, which consistently explains all the facts found in this study, including the observation of the $q$-dispersion for $d$-CT: it is the $t_{1 u}$ orbitals hybridizing with the $d$ orbitals in the adjacent clusters as mentioned in a previous report [2]. This extended cluster model is also supported by our tentative calculations on a larger $S=1,\left(\mathrm{Ni}^{-} \mathrm{O}_{6^{-}}\right.$ $\left.\mathrm{Ni}^{\prime} 12\right)^{10-}$ cluster $^{2}$. The calculation showed the $t_{1 u}$ to be strongly hybridized with the outside $\mathrm{Ni}$, exhibiting charge populations $\sim 50 \%$ in the $\mathrm{O}$ sites and $\sim 50 \%$ in the Ni sites. The $(\mathrm{B}) \rightarrow(\mathrm{A})$ dipolar transition hence lead to a substantial amount of $\mathrm{Ni}_{\text {out }} \rightarrow \mathrm{Ni}_{\text {in }}$ charge transfers. This argument originally arises from the experimental fact that the Mott-gap is defined by the dipolar transition, unlike the conventional charge transfer excitation having a nondipolar nature. Truly, our data and analysis themselves do not clarify whether or not the orbital (B) involves the $d$-components. Nevertheless, if they are assumed to be involved as suggested by other reports, then we believe that their contribution would be from $\mathrm{Ni}$ in adjacent clusters rather than $\mathrm{Ni}$ in the cluster. Again, the inter-cluster charge transfer (for the dipolar transition) and the intracluster charge transfer (for the non-dipolar one) would be reasonably linked with another experimental fact, i.e., the observation of a large $q$-dispersion for the former whereas the indiscernible dispersion for the latter.

Finally, we mention the relationship between our UHF molecular orbitals and the theory previously reported [9-13]. Those theoretical studies generally report (i) the existence of the UHB and the LHB derived from the $e_{g}$ orbitals, which are substantially separated in energy, (ii) the oxygen $p$ bands residing between the UHF and the LHB, partly hybridized with $d\left(e_{g}, t_{2 g}\right)$ bands, and most importantly, (iii) the well-hybridized $d-p$ bands at the top of the valence bands. The HF scheme generally reproduces the UHB and the LHB by the exchange split of the $e_{g}$ bands $[29,30]$. This is also the case of our calculation: the $e_{g}$ orbitals for up-spins have significantly low energy so that they position below the oxygen $p$ orbitals

${ }^{2}$ The calculation was made on a cluster having the nominal configuration of $\left(d \uparrow^{5} \downarrow^{3}-p^{6}-d \uparrow^{5} \downarrow^{5}\right)$. This configuration is much more easily converged than $\left(d \uparrow^{5} \downarrow^{3}-p^{6}-d \uparrow^{3} \downarrow^{5}\right)$ having an antiferromagnetic coupling, which is nearer the actual situation. 
(not shown), consequently reproducing the features-(i) and -(ii). The feature-(iii) can be associated with the oxygen $t_{1 u}$ molecular orbitals, assuming our model, i.e., the inter-cluster $e_{g}-p$ hybridization is valid. We should also mention that our energy diagram is similar with that of the $\mathrm{LDA}+\mathrm{U}$ method, where $U=8 \mathrm{eV}$, by Bengone et al. [31].

\section{Conclusions}

Electronic excitations in TMO were investigated by IXS and optical reflectivity measurements. The $q$-dependent dielectric functions of high quality were obtained from the experimental data through the Kramers-Kronig transformation. Two types of charge-transfer excitations, namely the dipolar and the non-dipolar charge transfer excitations, were identified. The former defines the Mott-gap (at $\sim 5 \mathrm{eV}$ ) showing a discernible $q$-dependence of its energy whereas the latter appears at higher energy (at $8 \sim 12 \mathrm{eV}$ ) without a dispersion. Comparison with the analyses using the molecular orbitals indicated that the dipolar charge transfer likely involved a substantial amount of charge transfer from TM to TM, in agreement with recent reports in $\mathrm{NiO}$, showing that the top of the valence band had TM $d$ portions as much as $\mathrm{O} p$ portions. These features were commonly observed for TMO of NaCl-type. On the other hand, the $d d$ excitations observed in $\mathrm{NiO}$ and $\mathrm{CoO}$ at low energies $(<4 \mathrm{eV})$ did not appear in $\mathrm{FeO}$ and $\mathrm{MnO}$. Those are attributed to an additional band near Fermi level presumably arising from the defects, and the half-filled electronic configuration in $\mathrm{FeO}$ and $\mathrm{MnO}$, respectively.

The experiments were performed in beam time approved by Japan Synchrotron Radiation Research Institute, SPring-8 (C05A12XU-1512N) and National Synchrotron Radiation Research Center, Taiwan (2005-2-005). We thank C.-C. Kao, G.A. Sawatzky, A. Baron, and T. Nomura for useful discussions.

\section{References}

1. A. Fujimori, F. Minami, S. Sugano, Phys. Rev. B 29, $5225(\mathrm{R})(1984)$

2. A. Fujimori, F. Minami, Phys. Rev. B 30, 957 (1984)

3. G.A. Sawatzky, J.W. Allen, Phys. Rev. Lett. 53, 2339 (1984)

4. J. Zaanen, G.A. Sawatzky, J.W. Allen, Phys. Rev. Lett. 55, 418 (1985)

5. A. Fujimori, N. Kimizuka, M. Taniguchi, S. Suga, Phys. Rev. B 36, 6691 (1987)

6. R.J. Lad, V.E. Henrich, Phys. Rev. B 39, 13478 (1989)

7. S.-P. Jeng, R.J. Lad, V.E. Henrich, Phys. Rev. B 43, 11971 (1991)
8. M. Takahashi, J.-I. Igarashi, Phys. Rev. B 54, 13566 (1996)

9. R. Eder, A. Dorneich, H. Winter, Phys. Rev. B 71, 045105 (2005)

10. X. Ren, I. Leonov, G. Keller, M. Kollar, I. Nekrasov, D. Vollhardt, Phys. Rev. B 74, 195114 (2006)

11. R. Eder, Phys. Rev. B 76, 241103(R) (2007)

12. J. Kuneš, V.I. Anisimov, S.L. Skornyakov, A.V. Lukoyanov, D. Vollhardt, Phys. Rev. Lett. 99, 156404 (2007)

13. Q. Yin, A. Gordienko, X. Wang, S.Y. Savrasov, Phys. Rev. Lett. 100, 066406 (2008)

14. M. Taguchi, M. Matsunami, Y. Ishida, R. Eguchi, A. Chainani, Y. Takata, M. Yabashi, K. Tamasaku, Y. Nishino, T. Ishikawa, Y. Senba, H. Ohashi, S. Shin, Phys. Rev. Lett. 100, 206401 (2008)

15. R.J. Powell, W.E. Spicer, Phys. Rev. B 2, 2182 (1970)

16. L. Messick, W.C. Walker, R. Glosser, Phys. Rev. B 6, 3941 (1972)

17. I. Balberg, H.L. Pinch, J. Mag. Mag. Mat. 7, 12 (1978)

18. B.C. Larson, W. Ku, J.Z. Tischler, C.C. Lee, O.D. Restrepo, A.G. Eguiluz, P. Zschack, K.D. Finkelstein, Phys. Rev. Lett. 99, 026401 (2007)

19. C.C. Kao, W.A.L. Caliebe, J.B. Hastings, J.M. Gillet, Phys. Rev. B 54, 16361 (1996)

20. H. Ishii, Y. Ishiwata, R. Eguchi, Y. Harada, M. Watanabe, A. Chainani, S. Shin, J. Phys. Soc. Jpn 70, 1813 (2001)

21. Y.Q. Cai, P. Chow, C.C. Chen, H. Ishii, K.L. Tsang, C.C. Kao, K.S. Liang, C.T. Chen, AIP Conference proceedings 705, 340 (2004)

22. K. Fukui, H. Nakagawa, I. Simoyama, K. Nakagawa, H. Okamura, T. Nanba, M. Hasumoto, T. Kinoshita, J. Synchrotron Rad. 5, 836 (1998)

23. M.W. Haverkort, A. Tanaka, L.H. Tjeng, G.A. Sawatzky, Phys. Rev. Lett. 99, 257401 (2007)

24. M.W. Schmidt, K.K. Baldridge, J.A. Boatz, S.T. Elbert, M.S. Gordon, J.H. Jensen, S. Koseki, N. Matsunaga, K.A. Nguyen, S.J. Su, T.L. Windus, M. Dupuis, J.A. Montgomery, J. Comput. Chem. 14, 1347 (1993)

25. W. Schülke, U. Bonse, H. Nagasawa, A. Kaprolat, A. Berthold, Phys. Rev. B 38, 2112 (1988)

26. L.C. Duda, T. Schmitt, M. Magnuson, J. Forsberg, A. Olsson, J. Nordgren, K. Okada, A. Kotani, Phys. Rev. Lett. 96, 067402 (2006)

27. G. Ghiringhelli, M. Matsubara, C. Dallera, F. Fracassi, A. Tagliaferri, N.B. Brookes, A. Kotani, L. Braicovich, Phys. Rev. B 73, 035111 (2006)

28. S.G. Chiuzbăian, G. Ghiringhelli, C. Dallera, M. Grioni, P. Amann, X. Wang, L. Braicovich, L. Patthey, Phys. Rev. Lett. 95, 197402 (2005)

29. M. Takahashi, J. Igarashi, T. Nomura, Phys. Rev. B 75, 235113 (2007)

30. S. Suga, S. Imada, A. Higashiya, A. Shigemoto, S. Kasai, M. Sing, H. Fujiwara, A. Sekiyama, A. Yamasaki, C. Kim, T. Nomura, J. Igarashi, M. Yabashi, T. Ishikawa, Phys. Rev. B 72, 081101(R) (2005)

31. O. Bengone, M. Alouani, P. Blöchl, J. Hugel, Phys. Rev. B 62, 16392 (2000) 